\title{
Moyamoya Disease in an 8-Year-Old Boy : Direct Bypass \\ Surgery in a Province of Peru
}

\section{Choque-Velasquez, Joham}

2017-12

Choque-Velasquez, J , Colasanti , R , Kozyrev, D A , Hernesniemi , J \& Kawashima , A 2017 , ' Moyamoya Disease in an 8-Year-Old Boy : Direct Bypass Surgery in a Province of

Peru ', World Neurosurgery , vol. 108 , pp. 50-53 . https://doi.org/10.1016/j.wneu.2017.08.112

http://hdl.handle.net/10138/298145

https://doi.org/10.1016/j.wneu.2017.08.112

publishedVersion

Downloaded from Helda, University of Helsinki institutional repository.

This is an electronic reprint of the original article.

This reprint may differ from the original in pagination and typographic detail.

Please cite the original version. 


\title{
Moyamoya Disease in an 8-Year-Old Boy: Direct Bypass Surgery in a Province of Peru
}

\author{
Joham Choque-Velasquez ${ }^{1,2}$, Roberto Colasanti, ${ }^{1,2,4}$, Danil A. Kozyrev², Juha Hernesniemi ${ }^{1,2}$, Akitsugu Kawashima ${ }^{1,3}$
}

\author{
BACKGROUND: Pediatric moyamoya cases may be very \\ arduous, even more so in a developing country, where \\ access to specialized centers may be prevented by \\ different factors.
}

CASE DESCRIPTION: Herein we report a challenging case, which was managed in the new Neurosurgical Center of Trujillo, regarding the direct anastomosis between the left superficial temporal artery and a cortical branch of the left middle cerebral artery in a 8-year-old Peruvian boy with moyamoya disease. Postoperatively, the patient's motor deficits and aphasia improved. To the best of our knowledge, this is the first performance of a direct revascularization for a pediatric moyamoya case in Peru.

CONCLUSIONS: The creation of highly specialized neurosurgical centers in the main strategic places of developing countries may allow optimal treatment of neurosurgical patients with complex diseases.

\section{INTRODUCTION}

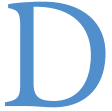

irect bypass surgery for moyamoya disease is particularly demanding, even more so in a developing country, where access to specialized centers may be impeded by different factors. The absence of neurosurgeons with specific experience in bypass procedures for pediatric moyamoya disease makes it practically impossible to use direct revascularization for these cases. Moreover, as previously reported, economic issues, an inequitable geographic distribution of major specialty services, and some cultural aspects, such as a large variety of native languages, contribute to exacerbate the disparities in the quality of health care between rural and urban populations in Peru. ${ }^{\mathrm{I}-4}$

In 2013, Es-Salud (Peruvian Social Security Health System) started a crucial plan called "Plan Confianza" with the aim to improve the aforementioned problems. This project promotes the development of specialized neurosurgical centers in the main strategic places all around the country to make feasible an optimal treatment of neurosurgical patients. The creation of a Neurosurgical Center of Excellence in Trujillo, led by Professor Juha Hernesniemi and his team, falls within this plan. 4,5

Herein we present a challenging case, which was managed in the new Neurosurgical Center of Trujillo, regarding the direct anastomosis between the left superficial temporal artery and a cortical branch of the left middle cerebral artery in an 8-year-old Peruvian boy with moyamoya disease. To the best of our knowledge, this is the first performance of direct revascularization for a pediatric moyamoya case in Peru.

\section{CASE REPORT}

A right-handed 8-year-old Peruvian boy was admitted to his local hospital in El Callao for recurrent right-sided tonic-clonic seizures associated with motor deficits and partial language deficit. His pathologic anamnesis was unremarkable except for a 4 -year history of chronic headache. A computed tomographic (CT) scan revealed diffuse cerebral edema in the left frontal region. Owing to the absence of angiographic studies, viral encephalitis was initially suspected, and empiric antiviral therapy was started, together with antiepileptic drugs.

Twenty days after the first admission, the patient again had tonic-clonic seizures associated with severe left-sided motor deficit. Moreover, his speech deficit reappeared and was more serious than the first time. During the next 48 hours the patient experienced severe neurologic deterioration and was transferred to the intensive care unit.

\section{Key words \\ - Bypass \\ Developing countries \\ - Direct revascularization \\ - Microneurosurgery \\ - Pediatric moyamoya disease}

From the ${ }^{1}$ Neurosurgical Unit, Es-Salud Trujillo Hospital, La Libertad, Peru; ${ }^{2}$ Department of Neurosurgery, Helsinki University Central Hospital, Helsinki, Finland; ${ }^{3}$ Department of Neurosurgery, Tokyo Women's Medical University, Tokyo, Japan; and ${ }^{4}$ Department of Neurosurgery, Umberto I General Hospital, Università Politecnica delle Marche, Ancona, Italy

\author{
To whom correspondence should be addressed: Joham Choque-Velasquez, M.D. \\ [E-mail: johchove@hotmail.com] \\ (-) Supplementary digital content available online. \\ Citation: World Neurosurg. (2017) 108:50-53. \\ http://dx.doi.org/10.1016/..wneu.2017.08.112 \\ Journal homepage: www.WORLDNEUROSURGERY.org \\ Available online: www.sciencedirect.com \\ 1878-8750/\$ - see front matter ๔ 2017 Elsevier Inc. All rights reserved.
}


Then, the patient was moved to a neurologic center of higher complexity in Lima, where he underwent further diagnostic studies. A cerebral CT scan and magnetic resonance imaging (MRI) evidenced acute ischemia in the right middle cerebral artery and right anterior cerebral artery territories. Cerebral digital subtraction angiography revealed complete occlusion of both internal carotid arteries, distal to the ophthalmic artery on the right side, and distal to the posterior communicating artery on the left side. Basal ganglia moyamoya collaterals were observed bilaterally. The right posterior communicating artery was patent after the injection of the contralateral left internal carotid artery; however, no significant collateralization was evident, particularly from the right external carotid artery (Figure 1).

Owing to the absence of neurosurgeons with experience in bypass procedures for pediatric moyamoya disease, it was decided to schedule an indirect revascularization procedure for the following months. Meanwhile, the patient's neurologic status partially improved, and he experienced severe left hemiparesis and expressive aphasia.

Since February 20I6, thanks to the cooperation of the Helsinki Neurosurgery Team and Es-Salud Peru, a high center of excellence in microneurosurgery has been developed in Trujillo, Peru. As a part of our activities, the "first cerebral bypass and vascular microsurgery live course" was held.

The patient underwent 2 direct bypass procedures between the left superficial temporal artery and the left middle cerebral artery, which were performed by the senior author (A.K.) (Figure 2). The postoperative course was uneventful, and surprisingly the patient could articulate some simple words at the immediate postoperative evaluation. One year after his surgery, the patient can perform his daily activities with some assistance, he is able to walk without any support, and he can pronounce simple words, still with some difficulty; Video 1 shows preoperative and postoperative radiologic studies and the postoperative neurologic status of the patient. However, a moderate left-side hemiparesis with severe distal dysfunction is still present. It may still be possible to perform an indirect or direct revascularization if a bypass surgeon is available for this young patient.

\section{DISCUSSION}

Economic, cultural, and geographic reasons usually limit the access to specialized health centers in developing countries, especially in rural areas. ${ }^{\mathrm{I}-4}$

In Peru, a country with a population of approximately 30 million, the most developed neurosurgical centers are in Lima, the capital of the country. The few neuroendovascular units of the country are also concentrated in the capital district.

Because the system for transferring patients does not work effectively, the transport of an inpatient from more than a thousand of kilometers far inside the country to Lima implies a huge monetary investment and may represent a high risk for the inpatient himself or herself. As described in our previous report, the benefits of centralized systems constitute a more simple protocol for the treatment of neurosurgical patients. Few neurosurgical centers assemble the best technologic resources and highly specialized surgical and diagnostic skills. The resulting high volume of specialist procedures in a few centers makes it easier and quicker to train of doctors, thus improving the quality of neurosurgical care. Obviously, centralized systems require an impeccable system for transferring patients. $^{4}$

On the other hand, as other experiences have demonstrates, ${ }^{6-8}$ we believe that an effective decentralization of major specialist services, together with an efficient use of the available resources, may allow the improvement of healthcare systems in developing countries. In fact, a wise decentralization could help distribute and retain highly specialized health workers in remote areas, thus ameliorating treatment outcomes in rural populations. As a result, this could make possible a straightforward management of most of the neurosurgical cases, and of all emergencies, while decreasing or limiting the transfer of patients to other centers just to the more complex and nonurgent cases. In addition, this could also permit the wait list for elective cases to be reduced while offering the entire population appropriate and well-timed clinical and surgical care. ${ }^{4,5}$

In May 20I6, thanks to the Plan Confianza program of Es-Salud, the first Peruvian Neurosurgical Center of Excellence was created in Trujillo. ${ }^{4,5,9-13}$ Moreover, thanks to the participation of prominent vascular neurosurgeons, the first cerebral bypass and vascular microsurgery live course was carried out. The senior author (A.K.) performed for the first time in Peru a left superficial temporal artery-to-middle cerebral artery bypass procedure in a pediatric patient with severe moyamoya disease.

A pediatric patient with severe moyamoya disease and bihemispheric symptoms is very challenging. Even though we initially planned an indirect revascularization, direct bypass surgery was finally carried out with the aim of reducing the risk of future ischemic complications in the relatively less damaged left cerebral hemisphere. Indeed, even if the brain MRI had not shown infarcts into the left language area, complete expressive aphasia was present. We believe that the impairment of language was related to a hypoperfusion resulting from narrow cerebral vessels in a still developing brain. Hence, we selected a direct revascularization procedure because of the limited left cerebral hemisphere ischemia in a patient with aphasia.

Initially, a simultaneous right side bypass was also considered. However, in the end, the plan of a simultaneous bilateral bypass was dismissed because of the high risk of hyperperfusion syndrome, postoperative hemorrhage, or both in a patient with a relatively recent large right cerebral hemisphere ischemia. At his Iyear follow-up visit, the clinical status of the patient is good and stable, and a new procedure for right direct or indirect revascularization is pending.

The diagnosis of moyamoya disease requires the evidence of narrowing of the distal internal carotid arteries, which extends to the proximal middle cerebral and anterior cerebral arteries, along with collateral circulation formation at the base of the brain. Cerebral angiography is the criterion standard for the diagnosis of moyamoya disease. ${ }^{\mathrm{I}, \mathrm{IS}}$

Class I recommendations for the treatment of moyamoya disease emphasize revascularization surgeries, which may 


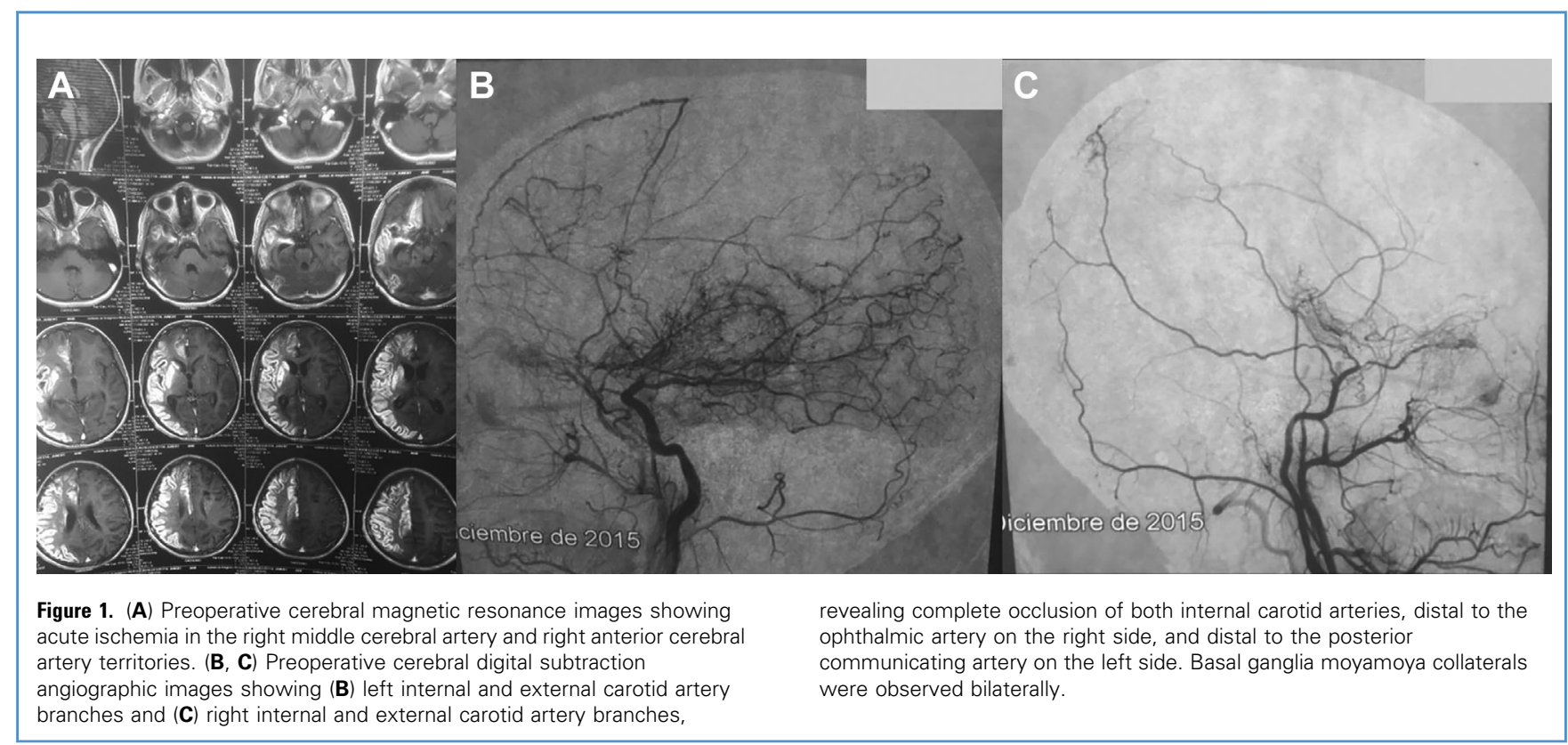

be categorized into direct and indirect procedures. ${ }^{16}$ Indirect revascularization is characterized by the placement of dura mater, temporalis muscle, or superficial temporal artery onto the surface of the brain to stimulate subsequent angiogenesis. Direct revascularization is usually mediated by the anastomosis between the superficial temporal artery and a cortical branch of the middle cerebral artery. This last procedure is challenging and demands an experienced vascular neurosurgeon. As a consequence, children with moyamoya disease have undergone indirect revascularization procedures in most cases. ${ }^{15}$
The senior author (A.K.), who has performed more than Iooo bypass procedures and has wide experience in direct bypass for moyamoya disease, decided to the perform superficial temporal artery-to-middle cerebral artery anastomosis on the basis of the above-mentioned clinical and radiologic features. ${ }^{\text {17-19 }}$

According to a recent systematic review, indirect revascularization is associated with superior long-term results compared with direct procedures in both children and adults. Moreover, possible complications related with direct bypass are hyperperfusion syndrome, probable rapid stenosis of the internal carotid artery, and disturbance in the development of moyamoya collaterals. ${ }^{20}$

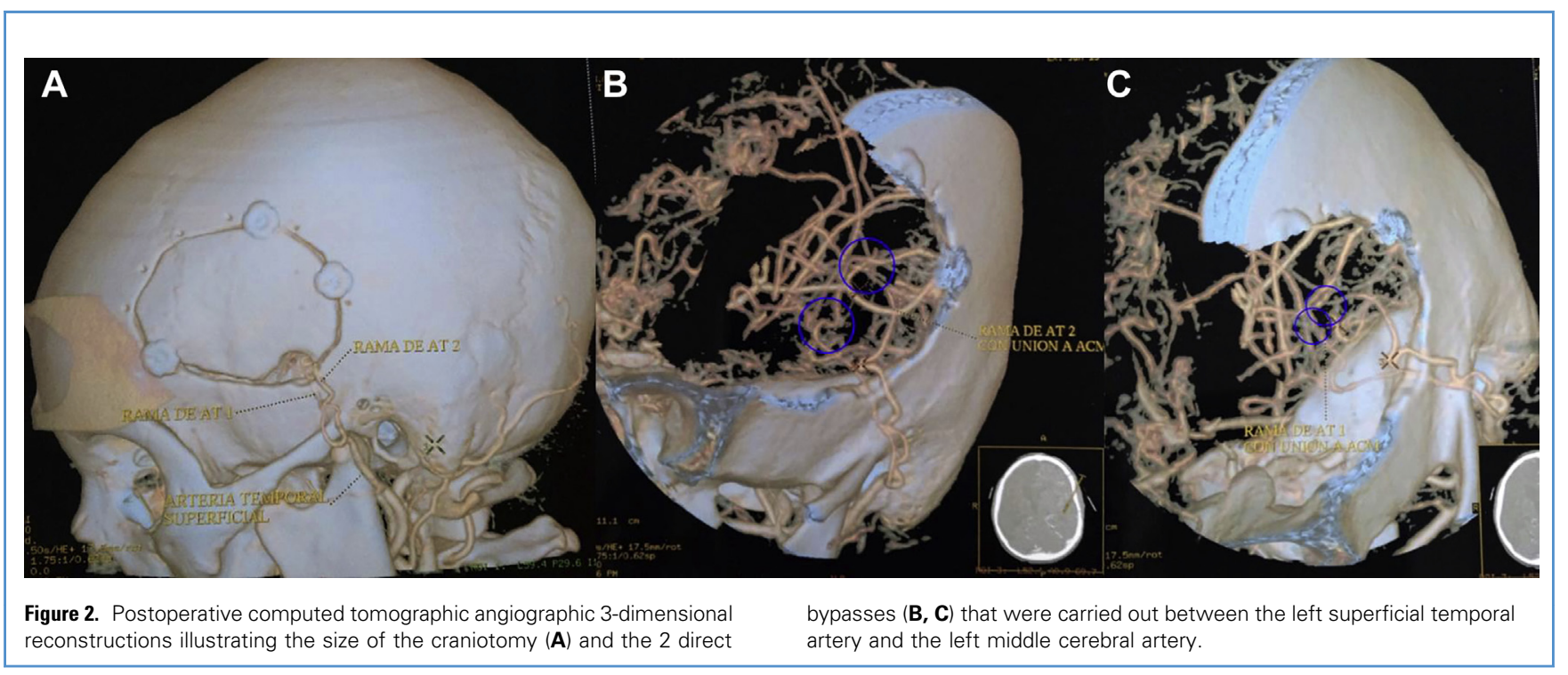


In addition, indirect techniques, which are technically less demanding, do not require temporary occlusion of the middle cerebral artery branches. Nonetheless, with indirect revascularization procedures, the development of collaterals is limited to the area of the craniotomy. ${ }^{2 \mathrm{I}}$ Moreover, indirect techniques take several months of angiogenesis, in contrast to the immediate reperfusion offered by a low-flow superficial temporal artery-tomiddle cerebral artery bypass. ${ }^{22}$

\section{CONCLUSION}

Bypass procedures for the treatment of moyamoya disease are particularly challenging in developing countries because of the necessity of a special environment and equipment. However, good teamwork coordination, a judicious application of microsurgical principles, and the presence of experienced vascular neurosurgeons may allow these surgeries to be performed with reliable results, even in resource-challenged environments.

\section{REFERENCES}

I. Behrman JR, Behrman JA, Perez NM. Out of sync? Demographic and other social science research on health conditions in developing countries. Demogr Res. 20II;24:45-78.

2. Camacho AV, Castro MD, Kaufman R. Cultural aspects related to the health of Andean women in Latin America: a key issue for progress toward the attainment of the Millennium Development Goals. Int J Gynaecol Obstet. 2006;94:357-363.

3. Larme AC. Health care allocation and selective neglect in rural Peru. Soc Sci Med 1982. 1997;44: I7II-I723.

4. Choque-Velasquez J, Colasanti R, Baffigo-Torre V, Sacieta-Carbajo LE, Olivari-Heredia J, FalconLizaraso Y, et al. Developing the first highly specialized neurosurgical center of excellence in Trujillo, Peru: work in progress-results of the first four months. World Neurosurg. 2017;I02: 334-339.

5. Choque-Velasquez J, Colasanti R, Fotakopoulos G, Elera-Florez H, Hernesniemi J. Seven cerebral aneurysms: a challenging case from the Andean slopes managed with I-stage surgery. World Neurosurg. 2017;97:565-570.

6. Adeleye AO, Fasunla JA, Young PH. Skull base surgery in a large, resource-poor, developing country with few neurosurgeons: prospects, challenges, and needs. World Neurosurg. 20I2;78: $35-43$.

7. Wahba MY, Kelly ME. The high cost of endovascular neurosurgery therapies and the need to sustain quality on limited resources. World Neurosurg. 20I4;82:7-8.

8. Davis MC, Than KD, Garton HJ. Cost effectiveness of a short-term pediatric neurosurgical brigade to Guatemala. World Neurosurg. 2014;82: 974-979.

9. Velasquez JC, Lau J, Kozyrev D, Sharafeddin F, Colasanti R, Luostarinen T, et al. Clean, fast and preserving normal anatomy: "the Helsinki revolution" in microneurosurgery. J Neurosurg Sci. 20I6;60:44-53.

Io. Choque-Velasquez J, Colasanti R, Jahromi BR, Hernesniemi J. "Squeeze maneuver" assisted by indocyanine green videoangiography: simple technique to "resuscitate" partially occluded bridging veins during microneurosurgical operations. World Neurosurg. 2017;97:225-230.

II. Choque-Velasquez J, Colasanti R, Jahromi BR, Rafei A, Sharafeddin F, Hernesniemi J. Shortburst bipolar coagulation for repairing partially damaged brain arteries preserving their flow: technical note. World Neurosurg. 2016;93:324-329.

I2. Sharafeddin F, Hafez A, Lehecka M, Raj R, Colasanti R, Rafiei A, et al. A5 segment aneurysm of the anterior cerebral artery, imbedded into the body of the corpus callosum: a case report. Surg Neurol Int. 20I7;8:I8.

I3. Choque-Velasquez J, Colasanti R, ResendizNieves JC, Jahromi BR, Kozyrev DA, Thiarawat P, et al. Supracerebellar infratentorial paramedian approach in Helsinki neurosurgery: cornerstones of a safe and effective route to the pineal region. World Neurosurg. 2017;105:534-542.

I4. Rhee JW, Magge SN. Moyamoya disease and surgical intervention. Curr Neurol Neurosci Rep. 20II; II:I79-I86.

15. Scott RM, Smith ER. Moyamoya disease and moyamoya syndrome. N Engl J Med. 2009;360: I226-I237.

I6. Roach ES, Golomb MR, Adams R, Biller J, Daniels S, Deveber G, et al. Management of stroke in infants and children: a scientific statement from a special writing group of the American Heart Association Stroke Council and the Council on Cardiovascular Disease in the Young. Stroke. 2008;39:2644-269I.

17. Kawashima A, Kawamata T, Yamaguchi K, Hori T, Okada Y. Successful superficial temporal artery-anterior cerebral artery direct bypass using a long graft for moyamoya disease: technical note. Neurosurgery. 2010;67:I45-I49 [discussion: I49].
I8. Okada Y, Kawashima A, Hori T. STA-MCA and STA-ACA anastomosis for moyamoya disease. No Shinkei Geka. 2009;37:959-971.

I9. Morisawa H, Kawamata $\mathrm{T}$, Kawashima $\mathrm{A}$ Hayashi M, Yamaguchi K, Yoneyama T, et al Hemodynamics and changes after STA-MCA anastomosis in moyamoya disease and atherosclerotic cerebrovascular disease measured by micro-Doppler ultrasonography. Neurosurg Rev. 20I3;36:4II-4I9.

20. Macyszyn L, Attiah M, Ma TS, Ali Z, Faught R, Hossain A, et al. Direct versus indirect revascularization procedures for moyamoya disease: a comparative effectiveness study. J Neurosurg. 20I7; I26:I523-I529.

2I. Kuroda S, Houkin K, Ishikawa T, Nakayama N, Ikeda J, Ishii N, et al. Determinants of intellectual outcome after surgical revascularization in pediatric moyamoya disease: a multivariate analysis. Childs Nerv Syst. 2004;20:302-308.

22. Houkin K, Nakayama N, Kuroda S, Ishikawa T, Nonaka T. How does angiogenesis develop in pediatric moyamoya disease after surgery? A prospective study with MR angiography. Childs Nerv Syst. 2004;20:734-74I.

Conflict of interest statement: Prof. Juha Hernesniemi is an Aesculap counselor. The C. Ehrnrooth Foundation partially supported the development of a high specialized neurosurgical center in the Es-Salud Trujillo hospital, La Libertad region, Perú. The remaining authors have no conflict of interest to report.

Received 8 July 2017; accepted 15 August 2017

Citation: World Neurosurg. (2017) 108:50-53. http://dx.doi.org/10.1016/j.wneu.2017.08.112

Journal homepage: www.WORLDNEUROSURGERY.org Available online: www.sciencedirect.com

1878-8750/\$ - see front matter (C) 2017 Elsevier Inc. All rights reserved. 